\title{
8 Europa im Vergleich: Ergebnisse einer kontrastiven Korpusanalyse
}

Anhand kontrastiver Textanalysen kann ermittelt werden, inwiefern die für den deutschen Diskurs typischen Kennzeichen verbal-antisemitischer Äußerungen auch generell charakteristisch für die Judenfeindschaft in Europa ${ }^{1}$ sind oder ob und inwieweit es (aufgrund der Spezifik der deutschen NS-Vergangenheit) signifikante Unterschiede gibt. Ein Subkorpus von 1.002 E-Mails und Briefen, die zwischen 2010 und 2011 an die Botschaften Israels in Wien, Bern, Den Haag, Madrid, Brüssel, London, Dublin und Stockholm geschickt wurden, ${ }^{2}$ ist dafür qualitativ und quantitativ untersucht worden. Quantitativ betrachtet lässt sich zunächst konstatieren, dass wesentlich weniger rechtsradikale Zuschriften zu verzeichnen sind. Während über 10 Prozent der deutschen Schreiber klar rechtsextremen und neonazistischen Gruppierungen zuzuordnen sind, ist der Anteil in den anderen europäischen Ländern deutlich geringer: Nur knappe drei Prozent der Verfasser sind Extremisten (mit der Ausnahme Österreichs, wo der Anteil bei über zehn Prozent liegt), der Großteil der Textproduzenten ist dagegen entweder in der Mitte der Gesellschaft oder stark links verortet. Quantitativ und qualitativ das signifikanteste Merkmal ist, dass nahezu alle Verfasser (d. h. 97 Prozent) die Formvariante des Anti-Israelismus kodieren, d. h. der Nahostkonflikt dient den Schreibern als Projektionsfläche für ihre judenfeindlichen Gedanken und Gefühle.

\section{Österreich}

Die größten Überschneidungen hinsichtlich Stereotypausdruck und judeophober Argumentation finden sich zwischen deutschen und österreichischen Verfassern: ${ }^{3}$ Hier sind auch die Tendenzen von Schuld- und Erinnerungsabwehr nahezu identisch, die bei den anderen Ländern nicht auftauchen (was an

1 Die aktuelle Antisemitismusforschung hat bislang Anzeichen für eine starke Verbreitung des anti-israelischen Antisemitismus festgestellt (s. hierzu u. a. die Beiträge in Faber et al. 2006 und Rensmann/Schoeps 2008). Empirische Umfragen belegen diese Tendenz (vgl. EUMC 2004, Zick/Küpper 2006a, b und Zick 2010). Zum anti-israelischen Antisemitismus in Amerika s. Reinharz (2010).

2 Es handelt sich hierbei um eine Auswahl von Schreiben, die über Stichproben ermittelt und aus denen ein Subkorpus erstellt wurde. Laut Auskunft des Botschaftspersonals in Paris, das ebenfalls wegen Materials angefragt worden war, werden seit 2010 kaum noch E-Mails oder Briefe an die Botschaft gesendet. Stattdessen veröffentlichen die Sprachproduzenten ihre (zahlreichen) kritischen Texte auf der Facebook-Seite der Botschaft.

3 Dies zeigt sich auch bei den zahlreichen Schmähbriefen, die von 2000 bis 2011 an die 
der engen historischen Symbiose dieser beiden Länder in der NS-Zeit und unter Umständen auch an der österreichischen Herkunft Hitlers liegt):
„Wenn die Politik der Israelis (Golan am Wochenende) und der Juden (immer dieses Rumgemecker und Hochhalten des Lebenselexiers Ausch- witz) so weitergeht, darf Israel und das Judentum sich nicht wundern, wenn es selbst dafür verantwortlich ist, dass es immer mehr Nazis produ- ziert - vielleicht gehöre ich auch bald dazu, denn langsam bin ich es auch leid!“ [IB_Wien_07.06.2011_Kun_001]

Eine starke Überdrussmentalität in Bezug auf die NS-Vergangenheit kommt in vielen österreichischen E-Mails wie in (1) zum Ausdruck. Diese ist zumeist gekoppelt an eine Täter-Opfer-Umkehr und das Klischee, Juden selbst erzeugten (durch angebliche Nazi-Methoden in Israel und Holocaust-Ausbeutung in Österreich) Antisemitismus. Entsprechend sind auf der sprachlichen Ebene Synonymverwendungen der Lexeme jüdisch und israelisch frequent. Zudem sind wie in (1) und (2) viele konzeptuelle Verschmelzungen von tradierten und aktuellen Stereotypen dominant.

(2) „Ich finde es nicht gut, dass die israelisch-jüdischen Toderschützen, wie der Jäger vom Hochsitz, gestern auf dem Golan vier, vielleicht sogar zwanzig Palästinenser wie wildes Vieh barbarisch und menschenverachtend, einfach der NS-Ideologie folgend, über den Haufen geschossen haben. Der besondere israelisch-jüdische Mut, in der restlichen Welt äußerste Feigheit genannt, wurde darin deutlich, dass man es fertig brachte, ein zwölfjähriges unbewaffnetes Kind mit schwerem militärischen Gerät und bewaffnet bis unter die Zähne einfach so abzuschlachten. Aber der ideologische Background ist offenbar die Thora, die solchen Ritualmord predigt.Einfach erbärmlich!“ [IB_Wien_06.06.2011_Kat_001]

Israel wird moralisch diskreditiert, indem judenfeindliche Stereotype, die vor allem der Dämonisierung dienen, reaktiviert und auf den jüdischen Staat projiziert werden.

Israelitische Kultusgemeinde in Wien sowie diverse jüdische Organisationen in Österreich gesendet wurden (und uns in einer komprimierten Zusammenfassung vorliegen). 


\section{Schweiz}

Schweizer Schreiber dagegen konzentrieren sich fast ausschließlich auf aktuelle Geschehnisse (im Nahen Osten) und zeichnen sich dabei durch eine extrem negative Haltung dem Staat Israel gegenüber aus, dessen Handlungen sie als unrechtmäßig, völkerrechtswidrig und verbrecherisch bezeichnen. De-realisierte Sachverhaltsdarstellungen und monokausale Schuldzuweisungen stehen im Vordergrund. Die E-Mail eines promovierten Baselers, der im Vorstand einer wirtschaftsorientierten Vereinigung ist, verdeutlicht dies:

„Nach den neuerlichen Massakern an 23 unbewaffneten Zivilisten, die
die Grenze zu den von Israel widerrechtlich besetzten Golanhöhen über-
schreiten wollten, werde ich darauf verzichten, Ihnen Vorträge über
Völker- und Menschenrecht zu halten, ... in der Schweiz leben ... viele
Flüchtlinge vor staatlichem Terrorismus wie ihn insbesondere auch
Israel praktiziert. ...Vielmehr kündet die Sprache der israelischen Regie-
rung von Xenophobie und Rassismus .......Sie können für diese Politik von
uns Bürgern weder Unterstützung noch Verständnis erwarten.“
[IB_Bern_23.05.2011_Die_001]

Konzeptuelle Gleichsetzungen von Israelis und Juden durch Synonymverwendungen sind vereinzelt ebenfalls zu beobachten:

(4) „Gebt dem Palstinensischen Volk endlich das gestohlene Land zurück. Die ganze Welt weiss, wie schändlich die Juden die Nachbarn behandeln!!!! Wir wünschen dem Palestinenischen Volk viel Erfolg bei der Gründung des eigenen Staates.“ [IB_Bern_24.09.2011_Por_001]

Viele Schweizer beteuern in ihren verbal-aggressiven E-Mails, dass sie keine Antisemiten seien, obgleich sie judeophobe Stereotype und verschwörungstheoretische Vorstellungen verbalisieren und in Analogie zur NS-Zeit zu Boykottaktionen aufrufen:

(5) „Ich habe nichts gegen die Juden generell, aber ich habe etwas gegen die wenigen extremen Juden und gegen einen Teil der israelischen Regierung, welche die ganze Welt in Schach halten und der Grund allen Übels in der Welt sind. ihr provoziert ja den Judenhass, den der grösste Teil der auf der Welt lebenden Juden natürlich nicht verdient. Aber wenn ihr den kleinen anderen Teil nicht unter Kontrolle halten könnt, natürlich mit der mächtigen Lobby ..., dann seid ihr eben auch schuld daran.“ [IB_Bern_16.10.2011_Wil_001] 
Die für Judenfeindschaft typische Widersprüchlichkeit zeigt sich: Einerseits ist die Artikulation höchst aggressiver Anschuldigungen und Drohungen zu sehen, andererseits die Leugnung ${ }^{4}$ und Marginalisierung von Antisemitismus als Motiv für diese praktizierte verbale Gewalt.

(6) „Dieses bescheidene Instrument, das ich besitze, nütze ich solange aus, als sich die Situation in den palästinensischen Gebieten nicht verbessert und ich fordere auch meinen Freundeskreis dazu auf, ebenfalls auf die Herkunft von Produkten aus Ihrem Land zu verzichten. Dieser Boykott ist nicht Ausdruck von anti-semitischen Gefühlen. Er soll Ausdruck sein, über einen Zustand, den ich als ungerecht und als zutiefst verachtenswert empfinde.“[IB_Bern_28.07.2011_Sto_001]

Insgesamt zeichnen sich alle Schreiben durch die Verwendung brachialer und unangemessener, da irreale Szenarien konstruierende Sprachgebrauchsmuster in Bezug auf Israel aus. Militäraktionen werden z. B. nahezu ausnahmslos als unnötige Massaker und als Ausdruck von Rassismus beschrieben.

\section{Niederlande}

Obgleich auch von den niederländischen Schreibern mehrheitlich ein gegenwartsbezogener, virulenter Israel-Hass kommuniziert wird, kommen vereinzelt vergangenheitsbezogene Verweise wie in (7) vor:

(7) „Ik kan hier kort over zijn: de verkeerde 6 miljoen zijn er vermoord toendertijd.Met de grootste minachting“ [IB_Den_Haag_12.07.2011_Cou_001] Deutsche Übersetzung:

„Das kann ich in aller Kürze sagen: Es wurden damals die falschen 6 Millionen umgebracht. Mit der größten Verachtung“

\footnotetext{
4 Der norwegische Schriftsteller Jostein Gaarder veröffentlichte am 5. August 2005 in der Zeitung Aftenposten den anti-israelischen Text Guds utvalgte folk, in dem er nahezu alle tradierten anti-jüdischen Stereotype auf Israel projizierte (, Wir nennen Kindermörder 'Kindermörder'... “; „, nicht die Spirale der Vergeltung der Blutrache von 'Auge um Auge, Zahn um Zahn'... ') und alle Strategien der Dämonisierung und Delegitimierung benutzte, um den jüdischen Staat zu diffamieren. Dennoch bestritt er vehement, einen antisemitischen Text produziert zu haben.
} 
Dies entspricht der in den deutschen Texten vielfach konstatierten konzeptuellen Kontrastierung GUTE JUDEN versus SCHLECHTE JUDEN (s. Kap. 5 und 11), wobei die ,guten Juden“ entweder tot oder extreme Israel-Kritiker sind, die ,schlechten Juden“ in Israel leben und/oder sich angeblich moralisch verwerflich benehmen. Die Implikatur in (7) ist aber nicht nur, dass es besser wäre, die jetzt in Israel lebenden Staatsbürger zu töten. Über die Anspielung auf den Holocaust wird auch impliziert, dass das Jüdisch-Sein der sechs Millionen entscheidend für die Verachtung und den Eliminierungswunsch des Verfassers ist.

Dominant sind in nahezu allen Schreiben aus den Niederlanden die Stereotype von Israelis als LANDRÄUBERN und SKRUPELLOSEN MÖRDERN SOwie die für den antisemitischen Anti-Israelismus charakteristischen Kriterien der De-Realisierung und Delegitimierung:

„When do you filthy murderers give the stolen land back to the rightfull people, the Palestinians! I despice all of Israël, this land has been stolen and now you are going to get on with murdering people who rightfully want THEIR land back, fuck all Israeli!“

[IB_Den_Haag_16.05.2011_Kpu_001]

\section{Spanien}

In den spanischen Schreiben werden besonders oft die Stereotype des Gottesmörders $^{5}$ und des ewigen Juden reaktiviert und kollektiv auf Israel projiziert: ${ }^{6}$

„Subject: Re: Activista 'Pacifico’ del Marmara. Esta fotografía fue publicada en la televisión turca.

5 Ein Grund hierfür könnte die stark katholische Ausrichtung der Gesellschaft sein, dies bleibt aber ohne weitere empirische Untersuchungen eine bloße Spekulation.

6 Auffallend bei dem spanischen Subkorpus ist aber, dass neben den zahlreichen hasserfüllten Schreiben auch ebenso viele positive E-Mails zu verzeichnen waren, in denen die Verfasser den virulenten Antisemitismus und Anti-Israelismus ihrer Landsleute und die vorverurteilenden Presseberichte in den Massenmedien beklagen und ihre solidarische Haltung mit Israel ausdrücken. Eine ähnliche Sachlage zeigt sich bei den Zuschriften aus Belgien anlässlich der Gaza-Flottillen-Aktion 2010: Bei der Botschaft in Brüssel gingen seinerzeit mehr positive Schreiben als negative ein. Viele Belgier schickten E-Mails, in denen sie betonen, keine Juden zu sein, aber die „pro-palästinensische Hysterie“ der belgischen Öffentlichkeit verurteilen. Vgl.: „Je crois heureusement ne pas etre le selu belge qui reprouve l'hysterie pro-palestienne...“ [IB_Brüssel_07.06.2010_Sim_001] („Ich glaube glücklicherweise nicht der einzige Belgier zu sein, der die pro-palästinensische Hysterie verurteilt..."). 
¡Dejarán ¡algun dia! de ser un pais perverso y criminal como cuando crucificaron a Cristo?Lo dudo. Siempre haciendo JUDIADAS. La única forma de de que dejaran de hacerlas serie meterles a todos, dejando a un lado a los poquitos judios decentes que quedarían , en parajes tan hospitalarios como treblinka o Auswich, Porque meterlos en el desierto de Nevada seria como llevarlos a una pasteleria. $\mathrm{Si}$ apareciese su tan esperado Mesías, uno nuevo que podia ser otro Cristo, ¿que harían con Él? lo probable es que le volverian a crucificar porque no se fian ni de su madre.“ [IB_Madrid_03.06.2010_Fer_001]

Deutsche Übersetzung:

„Betreff: Re: 'Friedens-'Aktivist von der Marmara. Dieses Photo wurde im türkischen Fernsehen veröffentlicht.

Werden Sie irgendwann! aufhören, ein ruchloses und kriminelles Land zu sein, wie damals, als Sie Christus gekreuzigt haben?! Ich bezweifle es. Sie werden immer JUDEREIEN begehen. Die einzige Möglichkeit, Sie davon abzuhalten, wäre, Sie alle, abgesehen von den wenigen anständigen Juden, die bleiben würden, in solch hospitalische Gegenden wie treblinka und Auschwitz zu schaffen, denn Sie in die Wüste von Nevada zu schicken, hieße, Sie in eine Konditorei zu führen. Wenn Ihr so sehr erwarteter Messias erscheint, ein neuer, der ein neuer Christus sein könnte, was würden Sie mit ihm machen? Wahrscheinlich würden Sie ihn erneut kreuzigen, weil Sie nicht einmal Ihrer Mutter vertrauen.“

NS-Vergleiche werden frequent benutzt; die Auslöschung des jüdischen Volkes wird als einzige Lösung für den Weltfrieden genannt:

(10) „Debo añadir que Vds. han superado en maldad y depravación a los nazis, pues además de ser tan malvados como ellos TODO lo manipulan hasta los extremos más increibles. Deben de ser expulsado de TODOS los lugares decentes pues son LArmoimn NEGACION DE TODO LO DIGNO Y deben ser tratados por lo menos, como tratan Vds a los palestinos, que los tratan como a escoria. Se deben, deberían, activar los campos de Auswich y Treblinka para volverlos a meter en ellos porque Vds. SON LA VERGÜENZA DE LA HUMANIDAD. No tienen perdón de Dios.Y USA, COMPAÑERO DE MISERIAS , quien siempre les ha protegido de las condenas de la humanidad, debe irse con Vds. a esos infames lugares de exterminio. Son lLA PURA VERGÜENZA DEL MUNDO.“

[IB_Madrid_03.06.2010_Jul_001]

Deutsche Übersetzung: 
„Ich muss hinzufügen, dass Sie die Nazis an Boshaftigkeit und Schändlichkeit übertroffen haben, und außerdem, dass Sie so bösartig wie jene sind. ALLES manipulieren Sie bis zu den unglaublichsten Extremen. Sie sollten von ALLEN ehrbaren Orten vertrieben werden, denn Sie sind DIE [unbekanntes Wort] NEGIERUNG VON ALLEM WÜRDIGEN UND Sie müssen mindestens so behandelt werden, wie Sie die Palästinenser behandeln, die Sie wie Abschaum behandeln. Man muss, müsste, die Lager von Auschwitz und Treblinka erneut eröffnen, um Sie wieder hinein zu schicken, weil Sie DER SCHANDFLECK DER MENSCHHEIT SIND. Gott verzeiht Ihnen nicht. UND DIE USA, WEGGEFÄHRTE DES ERBÄRMLICHEN, der Sie immer vor der Bestrafung durch die Menschheit beschützt hat, muss Sie in die schändlichen Vernichtungslager begleiten. Ihr seid DER SCHIERE SCHANDFLECK DER WELT.“

\section{Belgien}

Neben einigen kurzen vulgär-antisemitischen Zuschriften wie (11), in denen typische anti-jüdische Beschimpfungen mit dehumanisierender Semantik verbalisiert werden,

„Les Juifs sont des Merdes!“ [IB_Brüssel_01.07.2010_Tri_001] („Die Juden sind scheiße!")

überwiegen bei den belgischen Schreiben die als „humanistisch“ deklarierten und „aus Sorge geschriebenen“ anti-israelischen E-Mails, die in Israel mit seinen „scheußlichen Verbrechen“ und „Massakern“ („ces crimes innommables“, „les massacres") eine Gefahr für den Weltfrieden sehen:

„ils sont un danger pour la paix dans le monde...“

[IB_Brüssel_02.06.2010_Sam_001] (,sie sind eine Gefahr für den Weltfrieden...“)

Dabei werden jüdische und israelische Referenz-Ebenen vermischt sowie tradierte Stereotype (JUDEN SIND KEINE NORMAL EMPFINDENDEN MENSCHEN) verbalisiert, und der eliminatorische Antisemitismus von Vergangenheit und Gegenwart wird als begründet legitimiert:

(13) „Si vous étiez des Hommes, jamais vous ne re feriez vivre à d'autre le calvaire que vos parents ont vécu !!! C’est sa le judaïsme ? c'est faire du 
mal aux autres, c'est tuer des enfants et des femmes ? c'est bonbarder des bâteaux humanitaire? Alors je comprend mieux pourquoi on a voulu vous exterminer hier et aujourd'hui !!!“ [IB_Brüssel_31.05.2010_Sab_001] Deutsche Übersetzung:

„Wenn Sie Menschen wären, würden Sie niemals anderen den gleichen Leidensweg, den Ihre Eltern erlebt haben, zumuten! Ist das Judentum? Bedeutet es anderen weh zu tun, Kinder und Frauen umzubringen? Heißt es humanitäre Schiffe zu bombardieren? Wenn ja, dann verstehe ich besser, warum man Sie gestern und heute ausrotten wollte!“

\section{England}

Auffällig in den Texten aus England sind die zahlreichen monokausalen Schuldzuweisungen und die extremen Dämonisierungen Israels wie in (14), der E-Mail eines 60 Jahre alten Birminghamers, der expressis verbis bestätigt, dass seine Sicht in Bezug auf Israel allein auf sekundären Informationen basiert, d. h. auf Hörensagen und Berichten aus den Massenmedien:

(14) „I am listening on the radio to the ambassador. I am 60 years old and have been watching Israel over the past 50 year. My views have been informed by reading the history, talking to British soldiers who defended Palestine, and watching the activities of Israel. Israel does not have a right to exist. Its establishment after the the 2nd world war was a major mistake, and rode roughshod over the human rights of the residents of Palestine. People do not have the right to walk in and appropriate other people's land. The fact that some Hebrews lived there 2000 years ago is not relevant to the present. Until the current state of Israel accepts that it needs to return the land to the true owners of Palestine and abandons racial and religeous cleansing, it will be considered a pariah. The human cost has been and will be massive. Zionism has transformed from religeous philosophy to an evil world view.“ [IB_London_03.06.2011_Hoe_001]

Der Schreiber von (14) ist von der Wahrheit seines Glaubenssystems so unerschütterlich überzeugt, dass er ohne Bedenken kollektive Verurteilungen, antizionistische Beschuldigungen und abfällige Pauschaldelegitimierung artikuliert. Die Verknüpfung von jüdischen und israelischen Belangen wird durch den historischen Verweis „some Hebrews“ und die Entwertung von Zionismus als „evil world view“ indiziert. Sehr ähnlich ist die Argumentation eines akademischen 
Vielfachschreibers aus London, für den alle israelischen Militäraktionen prinzipiell „kriminell“ und „grundlos böse“ sind:

$$
\begin{aligned}
& \text { „All Israel achieves by these actions is to increase hatred against it and } \\
& \text { to further delegitimise itself as a civilised member of the international } \\
& \text { community.“ [IB_London_27.07.2011_Ale_001] }
\end{aligned}
$$

Israel wird kollektiv diskreditiert und von der zivilisierten Völkergemeinschaft ausgeschlossen, als „korruptes Apartheidsystem“ und „bankrottes Zionistenregime“ bezeichnet, das von „Betrügern, Dieben und Mördern“ beherrscht wird. Den Botschaftsangehörigen, die dem Schreiber geantwortet hatten, wird unterstellt, sie würden notorisch lügen (wobei er das tradierte Klischee von JUDEN ALS LÜGNERN UND BETRÜGERN bedient):

(16) „I don’t believe one word of your response. You lie, as always ..... It isn’t difficult for an army of occupation, operating under a corrupt apartheid legal system, to imprison whomever they like whenever they like for as long as they like. ... unfortunately for the Palestinian People it has had to wait some 44 years for a Godot to come and rescue it from the deadly grip of the morally bankrupt Zionist regime. In acting as an apologist for that gang of cheats, thieves and murderers you make yourself just as guilty as the worst racist settler in Hebron, throwing her garbage and worse onto the innocent men, women and children below.“

[IB_London_01.07.2011_Ale_001]

(17) ist die E-Mail eines promovierten Akademikers aus Oxford, der sich selbst als Pazifisten und Humanisten bezeichnet, der „angeekelt von den Taten der Israelis“ drastische explizite NS-Vergleiche („Israelis als Nazi-Abschaum“) verwendet, mit denen er alle Israelis kollektiv entwertet und diffamiert.

(17) „It is with disgust and horror that I watch the actions of Isreal. You behave like the Nazis, persecuting another race, committing atrocities. As a pacifist its hard to say this but I hope you pay the price according to your cred, namely an eye for an eye, I hope you suffer as much as the Palestinians (which would mean many many more Isrealis need to die to bring the justice you believe in). Disgusted human being who now sees Isrealis as Nazi scum. May your future pain bring humility.“

[IB_London_15.09.2011_Coo_001] 
Intensive Emotionen ausdrückende Lexeme (,with disgust and horror“) und Drohungen sowie Verwünschungen, die normalerweise mit einer pazifistischen Einstellung nicht vereinbar sind, kennzeichnen die extrem aggressiven Verbalhandlungen dieses Verfassers, der trotz seines Bildungsgrades tradierte judeophobe Klischees (,according to your cred, namely an eye for an eye“) bedient und sich der Tragweite der atavistischen und inhumanen Dimension seiner Argumentation (in der generische Leid- und Erniedrigungswünsche vorherrschen: „I hope you suffer“, „May your future pain bring humility“) nicht bewusst ist. Dass kognitive Haltung und emotionale Einstellung auseinanderklaffen, thematisiert der Schreiber zwar mit der Phrase „As a pacifist it is hard to say this, but...“, die adversativ den Widerspruch andeutet, doch ist das intensive Gefühl der Ablehnung und des Hasses größer als die vernunftgeleitete Überlegung. So wird durch die Semantik des Textes das übermächtige judenfeindliche Ressentiment transparent: Der kognitiv orientierte Pazifismus des Schreibers erstreckt sich nicht auf das jüdische Israel.

\section{Irland}

Eines der wenigen anonymen, vulgär-antisemitischen und sehr kurzen Schreiben, die lediglich dämonisierende Beschimpfungen und Verwünschungen enthalten, ohne argumentativ ins Detail zu gehen, stammt aus Irland:

$$
\begin{aligned}
& \text { „When I think of ISREAL I think of EVIL. I Hope you all Burn in Hell. } \\
& \text { Murdering SCUM you are } \underline{\underline{H} \text { ated }} \text { [dreifache Unterstreichung im Original, } \\
& \text { d. Verf.] all over the world. Stolen any IRISH PASSPORTS Lately??“ } \\
& \text { [IB_Dublin_10.06.2011_ano_001] }
\end{aligned}
$$

Die meisten Schreiber jedoch geben, wie die deutschen Textproduzenten auch, Namen und Adressen an, verlangen zum Teil Antworten und verfassen längere Texte, in denen sie pseudo-argumentativ ausführen, warum sie die Israelis bzw. Juden verachten und hassen. In (19), dem Brief eines Iren aus Westbury, verbinden sich so judeophobe Stereotypkodierungen mit anti-israelischen Diffamierungen:

(19) „It is with great anger that I put pen to paper so-as-to-speak to express my horror and utter disgust at the disgraceful behaviour of Israel [...] flotilla of Aid vessels bringing relief to an impoverished people, impoverished at the hands of a people which, I now see as the filth of the world. Israel has given the 'two fingers' to the decent and law abiding countries 
of the world and their peoples, effectively saying that as jews we can do what we like, we can murder who we like, we can abuse international laws, we can abuse passport systems, we can commit crimes 'when and where we like' and be answerable to nobody. There is little I can do. But, I can boycott all jewish products and businesses. ...It is now my considered opinion that the jews are in reality, an utterly despicable, vile (and a most horrid race of people.“[IB_Dublin_02.06.2010_Fit_001]

Tradierte, rassistisch basierte Stereotype (JUDEN ALS RASSE, JUDEN ALS AUSBEUTER UND NUTZNIESSER, JUDEN HABEN SONDERSTATUS) werden argumentativ in aktuelle, auf den Nahostkonflikt bezogene, anti-israelische Überlegungen eingebaut und als Begründung für das pseudo-kausale Gedankenfazit des Verfassers angeführt, dass Juden der Abschaum der Erde seien.

\section{Schweden}

Alle im Subkorpus analysierten Zuschriften zeichnen sich durch eine extreme Abwehrhaltung gegenüber Israel ${ }^{7}$ aus. Israel wird durchgängig als „Mörderstaat“ und „Unrechtssystem“ referenzialisiert und delegitimiert. Die negativen Gefühle werden auf die Botschaftsangehörigen übertragen; diese werden oft aufgefordert, das Land als unerwünschte Subjekte zu verlassen wie in (20), einer Zuschrift aus Schweden:

(20) „Still here? ? Get out or respect international laws. I will not go to Israel, please do not come here to my home country you killers Bye asap.“

[IB_Stockholm_20.07.2011_Tho_001]

Das Korpusmaterial zeigt auch eine europaweite, über die Landesgrenzen greifende Kommunikationspraxis gegen Israel, die durch die Internetkommunikation ermöglicht wird: Es gibt eine Reihe von Zuschriften, die am selben Tag mit identischem Wortlaut an verschiedene Botschaften Israels und an Regierungsstellen der betreffenden Länder sowie an zentrale Presseorgane gemailt werden. Dies geschieht teils durch einzelne Personen, teils durch israelfeindliche Gruppen, die miteinander vernetzt sind und ihre diffamierenden Handlungen koordinieren. So wurde beispielweise am 09.11.2011 ein und derselbe Text, der mit der Zeile in (21)

7 Zu den aktuellen Manifestationsvarianten von Antisemitismus in den skandinavischen Ländern s. Gerstenfeld (2008). 
beginnt, parallel an nahezu alle Botschaften Israels in Europa geschickt. Ausgetauscht wurden lediglich Anschrift und Namen der Absender.

„I am writing to you to register my disgust at the actions of the Israeli state and military in its treatment of the activists on board the MV Saoirse and Tahrir."

Entsprechende Kommunikationspraktiken sind auch im Internet in den sozialen Netzwerken zu beobachten. Die Globalisierung durch technischen Fortschritt wird von Antisemiten gezielt instrumentalisiert.

\section{Fazit}

Die für den judenfeindlichen Diskurs in Deutschland konstatierten Charakteristika und Trends stellen kein isoliertes Phänomen dar. Europaweit lassen sich in den Verbalmanifestationen ähnliche Merkmale feststellen. Neben der Artikulation $^{8}$ antisemitischer Stereotype ist insbesondere die zunehmende Kommunikation brachialer anti-israelischer Äußerungen $\mathrm{zu}$ beobachten. Die komparative Korpusstudie zeigt hier länderübergreifend eine große Übereinstimmung. Die für die Formvariante des Antisemitismus als Anti-Israelismus typischen Kennzeichen von De-Realisierung, Dämonisierung und Delegitimierung finden sich in allen Texten. Während sich jedoch die judenfeindliche Argumentation im aktuellen deutschen Diskurs durch die Verbindung von vergangenheitsbezogener Schuldabwehr und gegenwartsbezogener Schuldkonstruktion (mittels TäterOpfer-Umkehr) sowie durch die Projektion tradierter judenfeindlicher Stereotype auf Israel auszeichnet, dominiert in den antisemitischen Texten aus anderen europäischen Ländern klar die gegenwartsbezogene als Israel-Kritik artikulierte Judenfeindschaft. Wie in den deutschen Schreiben findet sich dabei allerdings auch oft konzeptuell und verbal die Verknüpfung bzw. Gleichsetzung von israelischen und jüdischen Aspekten. Die extreme Negativ-Konzeptualisierung von Israel wird entweder übergeneralisierend auf alle Juden übertragen oder umgekehrt werden tradierte judeophobe Stereotype ${ }^{9}$ auf Israel projiziert. So wie Juden

\footnotetext{
8 Dies korreliert mit Umfrageergebnissen innerhalb der Europäischen Union, die seit der Jahrtausendwende einen Anstieg antisemitischer Straftaten verzeichnet (s. Rensmann/Schoeps 2008: 10; s. z. B. die Ergebnisse der ADL 2007).

9 Somit decken sich die qualitativen Textanalysen nur bedingt mit den Ergebnissen von Umfragen. Zick/Küpper (2006b: 118) sehen als wesentlichen Trend im Meinungsbild der Europäer „eine Verschiebung von traditionellen antisemitischen Meinungen hin zu
} 
jahrhundertelang negativ konzeptualisiert und als Gegenentwurf zur Weltsicht der Betrachter ausgegrenzt wurden, so wird heute Israel als kollektiver Jude pauschal vorverurteilt, drastisch abgewertet und aus der Gemeinschaft der Völker bzw. Länder ausgeschlossen.

Auch bei gebildeten Schreibern lässt sich eine Einbettung des Stereotypengebrauchs in argumentative Muster beobachten. Wie in den deutschen Texten überwiegt eine Argumentation, die irrationale Radikalität und emotionale Aggressivität verbindet. Die vorherrschenden konzeptuellen Verschmelzungen sind JUDEN/ ISRAELIS $=$ TÄTER Und ISRAEL ALS VERBRECHERSTAAT $=$ JUDEN SIND VERBRECHER. Eine ausgeprägte Feindbildrhetorik gegenüber Israel weist dem jüdischen Staat die Rolle des Weltverbrechers zu. Der Nahostkonflikt wird nicht als ein vielschichtiges und kompliziertes Phänomen gesehen, sondern monokausal und zu Ungunsten Israels perspektiviert sowie auf eine simple, irreale Täter-OpferStruktur reduziert.

Während aber die Korpustexte aus Deutschland teils offensiv die emotionale Aggressivität, teils defensiv die implizite Schuld- und Erinnerungsabwehr zeigen, ist in den Schreiben der anderen Europäer klar die offensive Komponente dominant. Daher finden sich die Abschwächungs-, Legitimations-, Leugnungs- und Rechtfertigungsstrategien, die der moralischen Entlastung der Verfasser dienen sollen und die bei deutschen Schreibern (aus der Mitte) viel benutzt werden, um die radikale Semantik abzuschwächen und ein positives Selbstbild aufrecht zu erhalten, bei Verfassern aus anderen Ländern deutlich seltener. Auch die emotional fundierte Überdruss-Mentalität, die für viele deutsche Schreiber typisch ist und die sich in Scham- und Erinnerungsverweigerungen artikuliert, spielt bei den Textproduzenten aus anderen europäischen Ländern (mit der Ausnahme Österreichs) so gut wie keine Rolle.

subtilen, versteckten und sekundären antisemitischen Vorurteilen, die als transformierter Antisemitismus markiert" werden. Wir sehen jedoch die traditionellen Meinungen auch in den impliziten Verbalisierungsformen zum Vorschein kommen. Tradierte judeophobe Stereotype werden auf Israel projiziert (vgl. Kap. 7). So wird über die Umwegkommunikation zwar expressis verbis auf Israel referiert, dieses Referenzobjekt dann jedoch mittels aller gängigen judenfeindlichen Entwertungsmittel diffamiert. 